\title{
DIMENSIONAMENTO DA CAPACIDADE DE ATENDIMENTO UTILIZANDO SIMULAÇÃO A EVENTOS DISCRETOS: UM ESTUDO DE CASO NA LOTÉRICA LOTO LÍDER
}

\author{
Marcelo dos Santos Magalhães \\ Universidade Federal do Rio de Janeiro - UFRJ/Macaé \\ marcelomagalhaes@macae.ufrj.br \\ Louise Ferreira Gomes \\ Universidade Federal do Rio de Janeiro - UFRJ/Macaé \\ louise.gomes@ufrj.br
}

Isabella Arlochi de Oliveira

Universidade Federal do Rio de Janeiro - UFRJ/ Macaé

isabellaarlochi@hotmail.com

\section{RESUMO}

Localizada na principal avenida do centro comercial da cidade de Macaé, a Lotérica Loto Líder tem uma procura expressiva por seus serviços, principalmente no início de cada mês, provocando a formação de filas consideráveis. O longo tempo de espera despendido, além de inconveniente, faz com que 10 \% dos clientes desistam do serviço, causando prejuízo ao estabelecimento. Tendo em vista este fato a capacidade de atendimento, no período de maior demanda, é analisada através de dois modelos de simulação a eventos discretos, visando ao dimensionamento adequado do número de atendentes. Estes modelos foram desenvolvidos no programa Arena: o primeiro considera um sistema de atendimento de filas múltiplas e o segundo de fila única. Os resultados apresentados mostram que a capacidade de atendimento ideal é de quatro funcionários, sendo o sistema de fila única o melhor.

Palavras Chaves: Simulação; Teoria das Filas; Pesquisa Operacional

\begin{abstract}
Located on the main avenue of the commercial center of the city of Macaé, The Lotérica Loto Líder has an expressive demand for its services, mainly at the beginning of each month, provoking the formation of considerable queues. The long waiting time, besides inconvenience, causes that $10 \%$ of the customers give up of the service, causing damage to the establishment. Considering this fact, the service capacity, in the period of greatest demand, is analyzed through two models of simulation to discrete events, aiming at the adequate dimensioning of the number of attendants. These models were developed in the Arena program: the first considers a multiple queue attendance system and the second queue only. The results show that the ideal attendance capacity is four attendants; however, the single queue system is the most convenient.
\end{abstract}

Keywords: Simulation; Queue Theory; Operational Research 


\section{Como Citar:}

MAGALHÃES, Marcelo dos Santos; GOMES, Louise Ferreira; OLIVEIRA, Isabella Arlochi de. Dimensionamento da capacidade de atendimento utilizando simulação a eventos discretos: um estudo de caso na lotérica loto líder. In: SIMPÓSIO DE PESQUISA OPERACIONAL E LOGÍSTICA DA MARINHA, 19., 2019, Rio de Janeiro, RJ. Anais [...]. Rio de Janeiro: Centro de Análises de Sistemas Navais, 2019.

\section{INTRODUÇÃO}

Nos últimos tempos, os serviços vêm ganhando um destaque no cenário global, pois empregam mais que as fábricas e representam a maior parte do produto interno bruto (PIB) de muitos países (PETRÔNIO E LAUGENI, 2005). Todavia, os meios necessários à execução dos serviços, na maioria dos casos, não são suficientes para atender a demanda, provocando a formação de filas. Segundo Slack e et al. (2015) um sistema onde há existência de filas é resultado da indeterminação do tempo entre chegada de clientes e atendimento dos servidores.

Devido à intangibilidade dos serviços, a qualidade desta atividade é percebida com o nível de satisfação dos clientes e a fidelização dos mesmos. Estes dois aspectos, na maior parte dos ambientes, são reflexos do atendimento das perspectivas e do tempo de espera despendido nas filas. Estes fatos incentivam a busca por sistemas balanceados a um custo acessível por gestores de diferentes segmentos (DIAS E ET AL., 2017).

Gianese e Corrêa (1996) afirmam que no mundo contemporâneo existe a relação interpessoal direta ou indireta, envolvendo a obrigação de esperar. Para estes autores oferecer um serviço de qualidade significa compreender a demanda dos clientes, ajustar a capacidade de seus processos e, como consequência, reduzir o tempo de espera e aumentar o nível de satisfação dos usuários.

Para Prado (2014) um sistema é dito balanceado quando está adequadamente dimensionado, ou seja, os efeitos negativos do gargalo são reduzidos a um nível tolerável. Segundo este autor os gargalos são as atividades que registram o maior número de clientes em fila e existem duas técnicas para dimensionar adequadamente um sistema: a teoria das filas e da simulação. A primeira utiliza fórmulas matemáticas e a segunda um modelo representativo do sistema em um computador digital.

Outro aspecto constatado neste cenário são as penalidades impostas pela legislação de alguns Estados da Federação Brasileira aos estabelecimentos que proporcionem um tempo de espera acima de um determinado limite. Melo e et al. (2017) constataram que no Estado do Maranhão, por exemplo, existe uma lei que impõe aos estabelecimentos um tempo máximo de espera em fila de 30 minutos. Os prestadores de serviço que não cumprem esta meta estão sujeitos à advertência e multas no valor de $\mathrm{R}$ \$ 200 em caso de reincidência.

Neste contexto a Lotérica Loto Líder tem grandes desafios para prestar um serviço de qualidade a seus usuários. Localizada na principal avenida do centro comercial da cidade de Macaé, a demanda pelos serviços deste estabelecimento é expressiva, principalmente no início de cada mês, causando filas consideráveis. Além do transtorno, a longa espera causa prejuízo ao estabelecimento, pois $10 \%$ dos clientes desistem do serviço.

Tendo em vista os fatos expostos, o objetivo deste trabalho é dimensionar capacidade de atendimento da lotérica através da simulação de eventos discretos de forma que todos os clientes sejam atendidos e que o tempo máximo de espera não ultrapasse 30 minutos. Para isto serão desenvolvidos dois modelos de simulação no software Arena: o primeiro modelo considerará o sistema com múltiplas filas e o segundo com fila única.

Este artigo está estruturado em seis capítulos, incluindo esta introdução. Os capítulos dois e três abordarão a metodologia adotada e o referencial teórico. Os capítulos 
quatro e cinco o estudo de caso, a identificação do problema e a simulação do estudo de caso. O capítulo seis encerra o trabalho com a conclusão.

\section{METODOLOGIA}

Yin (2010) afirma que um estudo de caso é considerado uma pesquisa quando o pesquisador utiliza uma grande variedade de dados coletados. Segundo este autor as pesquisas podem ser quantitativas ou qualitativas. Prodanov e Freitas (2013) definem que uma pesquisa é quantitativa quando os fatos estudados são quantificados e que busca a relação causa e efeito do fenômeno analisado. Em oposição, a pesquisa qualitativa é aquela que não quantifica, ou seja, analisa apenas a relação do mundo real com o sujeito.

Para Da Silva e et al. (2016) as pesquisas são classificadas como: descritiva e explicativa; virtual e bibliográfica. A descritiva e explicativa detalha e discute os resultados quantificados do estudo. A virtual e bibliográfica é que utiliza livros, artigos e sites específicos para abordar um determinado tema.

Este trabalho realizou uma pesquisa quantitativa; descritiva e explicativa; virtual e bibliográfica para estudar a capacidade de atendimento da Lotérica. Através da pesquisa virtual e bibliográfica se desenvolveu as seções 3. A simulação do caso e análise dos resultados obtidos se baseou em uma pesquisa quantitativa, descritiva e explicativa.

\section{REFERENCIAL TEÓRICO}

Tendo em vista à larga utilização da simulação em diversos contextos, nesta seção serão apresentados os principais conceitos, assim como as vantagens, desvantagens e as contribuições de alguns trabalhos que utilizaram a técnica da simulação em diferentes áreas. Também serão apresentados os principais conceitos relacionados a um elemento existente em qualquer sistema: as filas de espera.

Kelton e Law (2000) definem a simulação como um processo de concepção de um modelo computadorizado de um sistema real para efeitos de testes experimentais, obtendo, deste modo, uma melhor compreensão do comportamento do sistema para um determinado conjunto de condições. Seguindo a mesma linha, Pegden (1995) enfatiza a facilidade de diagnosticar problemas ao se realizar uma simulação. Este autor aborda, também, como as variáveis podem ser deliberadamente manipuladas, para verificar, com clareza, como elas influem num determinado sistema em estudo.

Magalhães (2006) compreende a simulação como uma imitação de uma operação ou de um processo do mundo real, independente do uso de computadores. A simulação envolve a geração de uma história artificial de um sistema para análise de suas características operacionais. Este autor define sistema como um agrupamento de insumos e recursos para atingir um determinado objetivo, como por exemplo: uma fábrica, um hospital, uma escola, um posto de serviço e etc.

Freitas Filho (2008) compreende que os modelos são uma representação dos sistemas visando ao estudo dos mesmos, sendo classificados em contínuos e discretos. Os modelos discretos são aqueles nos quais as entidades são contáveis ou enumeráveis tais como: peças processadas em uma fábrica, clientes que chegam a um banco, a um refeitório e etc. Os modelos contínuos são aqueles em que as entidades não são enumeráveis, tais como: o nível de água de um reservatório, de um fluido em um duto e etc. Ainda segundo este autor as entidades são classificadas como dinâmicas ou estáticas. As dinâmicas são aquelas que se movem através do sistema e a estática são aquelas que servem outras entidades. Como exemplo de entidades dinâmicas, ele cita as peças que se movem em uma fábrica e os clientes que chegam e saem da fila do caixa de um supermercado. Como estática, menciona uma máquina (no caso da fábrica) e o caixa do supermercado. 
Para Magalhães (2006) a simulação é vantajosa quando ela imita o sistema real com menor custo ou menor quantidade de recursos. Os dados de saída da simulação devem corresponder diretamente às saídas que se obteriam no sistema real. Tendo em vista este fato, a técnica possibilita que novas políticas e procedimentos operacionais sejam estudados sem interferência direta no sistema, novos equipamentos e arranjos físicos sejam testados sem sua aquisição ou interferência direta na atividade e hipóteses sejam analisadas para que seja verificada sua praticidade.

Todavia, Freitas Filho (2008) cita as seguintes desvantagens da utilização da simulação: a construção dos modelos requer treinamento; os resultados da simulação podem ser difíceis de interpretar e, em alguns casos, a modelagem e análise da simulação podem consumir muito tempo e recursos. Mesmo com estes pontos negativo, este autor afirma que a aplicabilidade desta técnica é bastante expressiva, podendo ser percebida em diversos tipos de sistemas: manufatura; saúde; transporte; recursos naturais; construção; entretenimento e de processo de negócios.

Na área de serviço Melo e et al. (2017) usaram a simulação para dimensionar a capacidade de atendimento de uma agência dos correios, localizada em uma cidade do Estado do Maranhão, visando ao atendimento da legislação estadual 7806 de dezembro de 2002 que determina aos estabelecimentos uma espera máxima de 30 minutos sob pena de advertência e multa em caso de descumprimento. Estes autores constataram que os três funcionários destinados ao atendimento, no período de maior demanda (parte da manhã), não atingiam esta meta, pois o maior tempo de espera registrado foi de 33,5 minutos. Tendo em vista este fato, foi proposto que um funcionário interno fosse deslocado ao atendimento neste período. Esta alteração no modelo atendeu de maneira satisfatória a expectativa da organização, pois proporcionou um tempo máximo de espera de 11,4 minutos sem causar nenhum transtorno na prestação dos serviços.

Na área de educação, Dias e et al. (2017) constataram que a fila de aceso aos fornos de micro-ondas para aquecimento das refeições dos discentes da Universidade Federal do Rio de Janeiro é um problema crítico que prejudica o desempenho das atividades acadêmicas. Eles constataram que no local havia duas unidades deste recurso que provocava um tempo médio de espera superior a trinta minutos. Todavia, através da simulação de eventos discretos, eles constataram que o ideal seria a existência de quatro unidades deste recurso, pois o tempo de espera se reduziria para 16,83 minutos.

Na área de alimentação Magalhães e et al. (2017) simularam o processo de produção de uma pizzaria. Os autores constataram que o gargalo do processo era o forno que assava as pizzas. No local, havia um forno com capacidade para duas pizzas que no período de maior demanda provocava uma espera de 95,24 minutos. Através da simulação, constatou-se que a aquisição de um forno com capacidade para três pizzas reduziria este tempo para 45 minutos.

Na área militar, Praia e Gomes (2013) utilizaram a simulação para estudar o processo de recebimento de materiais do Sistema de Abastecimento da Marinha do Brasil. O estudo mostrou que o gargalo do processo é a atividade de perícia dos materiais, pois é a que apresenta o maior número de entidades aguardando na fila. $\mathrm{O}$ aumento de capacidade deste gargalo proporcionou uma redução de $95,9 \%$ no tempo de permanência no sistema e um aumento do número de itens processados de 23 para 47.

Segundo Prado (2014) uma das finalidades da simulação é a obtenção de um sistema balanceado. Um importante componente de um sistema são as filas, que também são definidas como sistemas. As filas são entendidas como um conjunto de entidades dinâmicas aguardando o atendimento por um determinado servidor. Na maioria das vezes, as filas são incômodas e refletem o nível de insatisfação dos clientes. Portanto, um sistema balanceado é aquele em que os efeitos negativos das filas são reduzidos a um nível tolerável. 
De acordo com Andrade (1990) um sistema de filas é caracterizado pelos seguintes elementos: distribuição de probabilidade do tempo de chegada dos clientes e de atendimento dos servidores; número de servidores; capacidade do sistema; tamanho da população e disciplina de atendimento. Este sistema é representado na Figura 1.

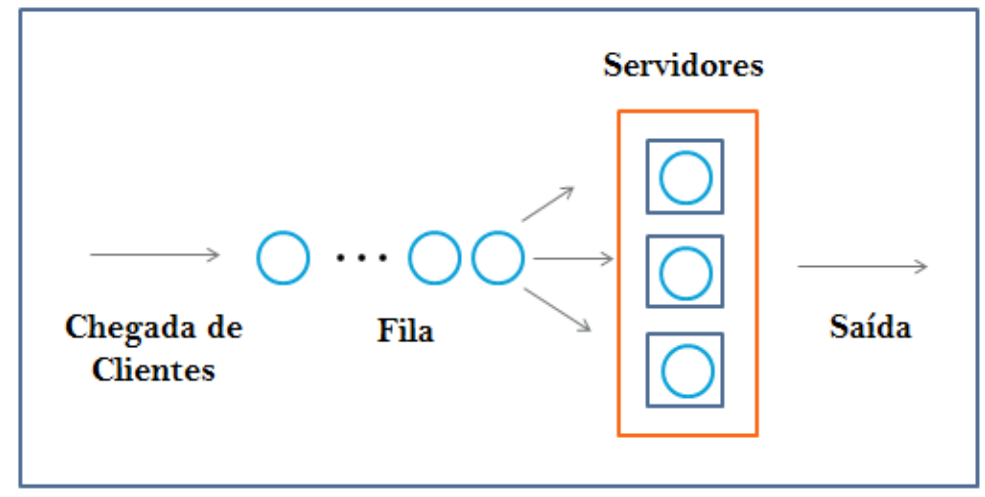

Figura 1 - Sistema de Filas. Fonte: Andrade (1990)

\section{ESTUDO DE CASO E IDENTIFICAÇÃO DO PROBLEMA}

Localiza em uma das principais avenidas do centro comercial do município de Macaé, situado na baixada litorânea do Estado do Rio de Janeiro, a lotérica Loto Líder, por ser credenciada a Caixa Econômica Federal, oferece aos seus usuários os serviços de pagamento de contas, depósitos, saques e jogos.

No trabalho de campo, observou-se que o estabelecimento funciona no período compreendido entre 8:00 da manhã e 17:00 da tarde e possui duas filas: a primeira destinada ao público com necessidades especiais (deficiência física ou mobilidade reduzida, idosos, gestantes, lactantes e pessoas com crianças de colo) e a segunda destinada para o restante dos clientes. Também existem, no estabelecimento, três funcionários para o atendimento. Um destes atendentes é exclusivo da fila preferencial, todavia, não havendo este perfil em fila ele pode atender aos demais casos. Os demais servidores são destinados ao público não preferencial.

No momento que o cliente entra na lotérica, ele escolhe em qual das filas se deslocará: preferencial ou na normal. Dependendo do tempo que o usuário dispenderá na fila, ele pode optar por permanecer no local e aguardar o atendimento ou desistir do serviço. Este fluxo é ilustrado na Figura 2.

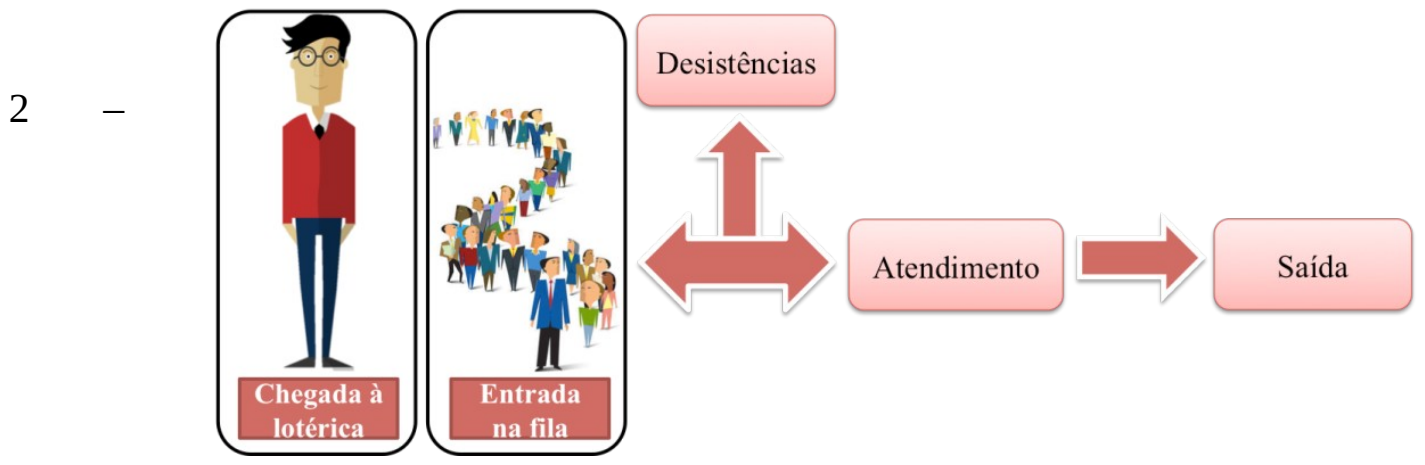

Sistema de Atendimento da Lotérica. Fonte: Própria 
Devido a sua localização privilegiada, a demanda pelos serviços desta instituição é expressiva e torna-se crítica, principalmente, no início de cada mês. Nas entrevistas realizadas com os funcionários e o proprietário da lotérica se constatou que $10 \%$ dos clientes desistem do serviço, neste período, devido à espera longa nas filas.

Tendo em vista este fato à primeira discussão que surgiu foi se seria mais viável adotar um sistema de atendimento com múltiplas filas, equivalente ao adotado pelos supermercados, ou um com fila única semelhante ao utilizado pelos bancos. Com isto, neste trabalho serão propostos dois modelos de simulação que dimensionarão a capacidade de atendimento do sistema de forma que não haja desistência de clientes.

Para isto, tendo em vista o trabalho de Melo e et al. (2017), estipulou-se que o maior tempo de espera não seja superior a 30 minutos em ambos os modelos. O primeiro analisará o sistema com múltiplas filas e o segundo com fila única. Em ambos os modelos não será feita a distinção entre público preferencial e não preferencial.

\section{SIMULAÇÃO DO ESTUDO DE CASO}

De acordo com Freitas Filhos (2008) um projeto envolvendo modelagem e simulação de sistemas só pode fornecer resultados adequados à tomada de decisão se os seguintes passos forem adotados: formulação e análise do problema, planejamento do projeto, formulação do modelo conceitual, coleta de dados, tradução do modelo, verificação e validação, projeto experimental final, experimentação, interpretação e análise estatística dos resultados, comparação de sistemas e identificação das melhores soluções, documentação, apresentação dos resultados e implementação.

Chwif e Medina (2010) simplificam estas etapas a três passos para que um trabalho de modelagem e simulação seja bem sucedido: concepção ou formulação do modelo, implementação do modelo e análise dos resultados. Os modelos propostos neste artigo seguirão esta metodologia.

A concepção ou formulação do modelo compreende entender claramente o sistema a ser simulado, a criação de um modelo conceitual e a coleta dos dados de entrada. As duas primeiras atividades deste passo foram realizadas na seção quatro.

A terceira atividade, coleta de dados, engloba a obtenção e o tratamento estatístico, visando à identificação da melhor distribuição de probabilidade que se ajusta aos mesmos. A coleta de dados foi realizada no início do mês de dezembro de 2017 na parte da manhã, período considerado de maior demanda pelos funcionários e proprietário. Foi registrado o tempo entre chegada e de atendimento de 60 clientes.

Uma vez obtidos estes dados, utilizou-se a ferramenta Input Analayser do Software Arena para verificar em qual distribuição teórica estes dados se ajustavam, com isto, se constatou que os tempos entre chegadas se ajustam a uma distribuição LOGN $(0.672,0.783)$. De forma análoga se constatou que os tempos de atendimento dos clientes se ajustam a expressão 1+ LOGN (1.24,1. 41).

O segundo passo da metodologia, implementação do modelo, compreende a conversão do modelo conceitual, obtido na seção 4, para um modelo computacional através de alguma linguagem de programação ou de um simulador comercial. Desta forma, realizou a construção do modelo de fila múltiplas no software Arena, usando os seguintes módulos lógicos do programa: create, process, enter, leave, pickstation e dispose.

No módulo create é definido a entidade principal (clientes) e a distribuição de probabilidade do tempo entre as chegadas. No process, são definidos os recursos (atendentes) e as distribuições dos tempos de atendimento. O módulo enter é equivalente ao station onde são definidas as estações de trabalho para animação. Neste modelo as estações definidas são: entrada; caixa um, dois, três, quatro, cinco e saída. No leave são definidos as 
rotas e os tempos de deslocamento entre as estações. Neste trabalho constatou-se que o tempo de deslocamento entre a entrada e os caixas é de cinco segundos e dos guichês para saída também é de cinco segundos.

O pickstation faz o modelo reproduzir o dinamismo de funcionamento de um sistema de múltiplas filas existente na maioria dos estabelecimentos. Os clientes, geralmente, dirigem-se aos caixas onde há o menor número de clientes em fila, ou o menor número de servidores ocupados ou menor número de clientes em rota. Nele também é definido o tempo de deslocamento dos clientes da entrada aos guichês. O módulo dispose finaliza o modelo. As configurações do módulo picksation são apresentadas na figura 3, o modelo lógico e a animação nas figuras 4 e 5.

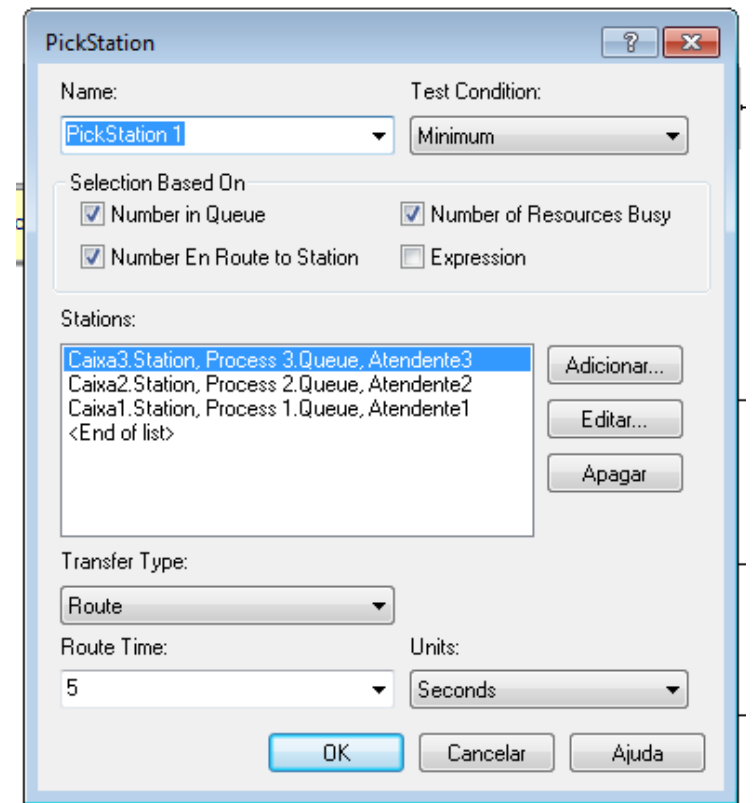

Figura 3 - Configuração do módulo pickstantion. Fonte: Simulador

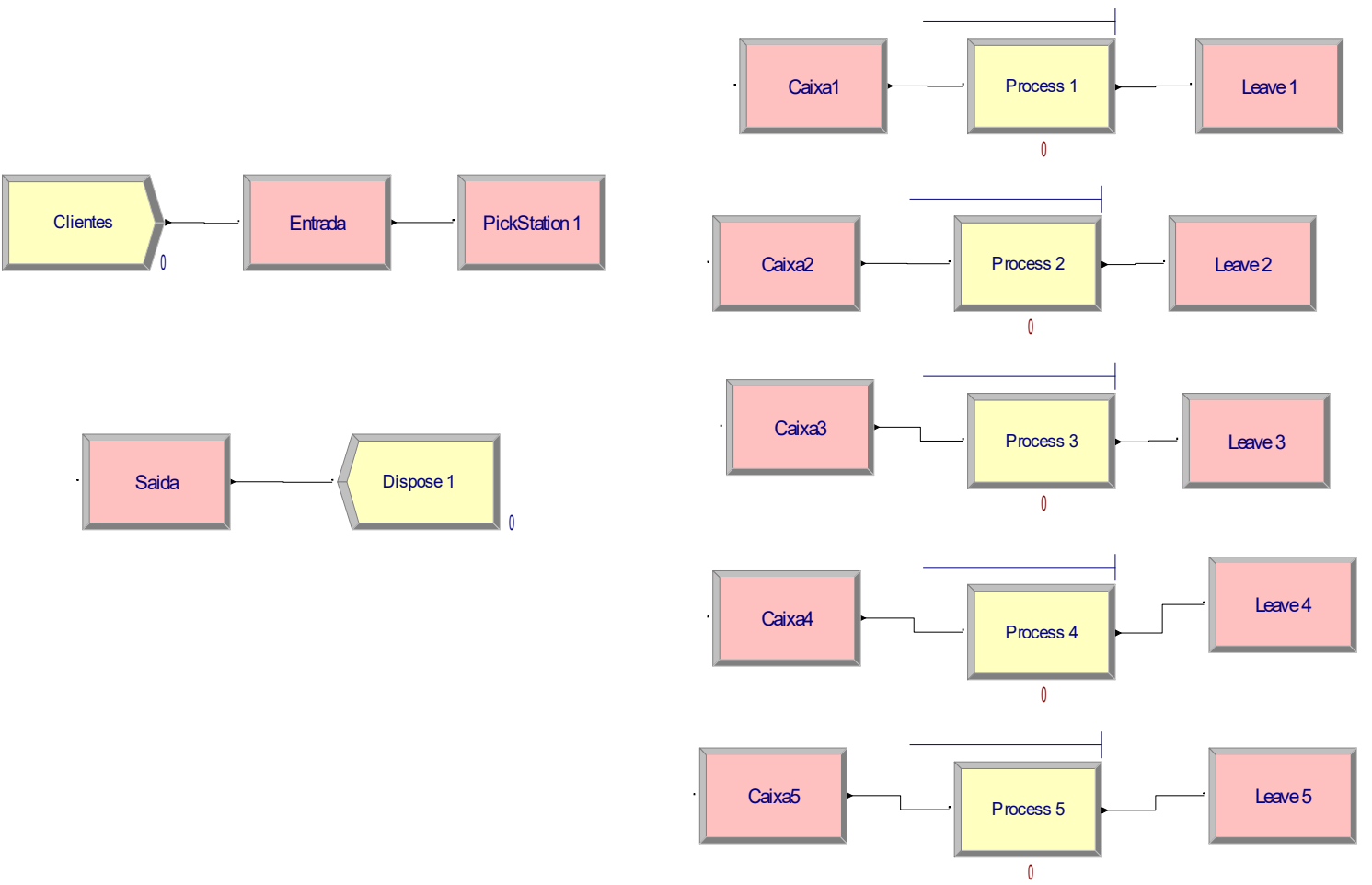


Figura 4 - Modelo Lógico do Sistema de Múltiplas Filas. Fonte: Simulador

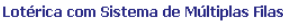

Relógio 16:00:00

Figura

$$
\begin{array}{r}
\text { Entradas } \\
\hline 08.00
\end{array}
$$

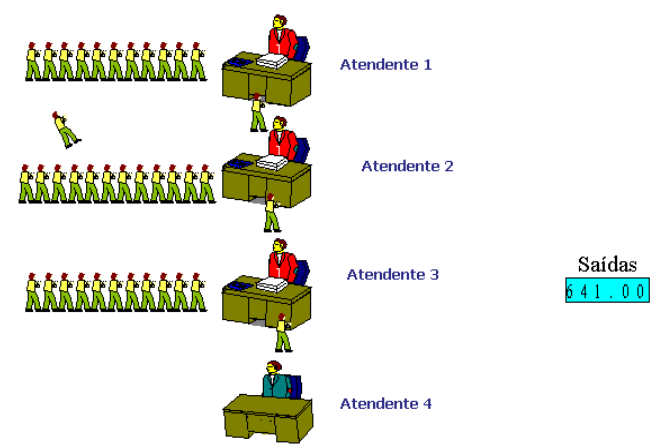

Atendente 5

Animação do Sistema de Múltiplas Filas. Fonte: Simulador

O modelo do sistema de fila única para mais de um servidor utilizou os mesmos módulos do anterior, incluindo o size e o release. O pickstation trabalha em conjunto com o size, todavia, ao contrário do primeiro modelo, definimos os critérios de seleção do usuário o menor número de servidores ocupados e o menor número em rotas para as estações. No size é definida a fila única e o recurso espaço. No pickstation, adicionamos ou retiramos caixas ao sistema. Todavia, para que o modelo funcione adequadamente a capacidade do recurso espaço deve ser equivalente a do pickstation. O release libera um cliente da fila para se dirigir ao caixa, quando este fica desocupado. Este modelo é representado na Figura 6 e a animação na Figura 7.
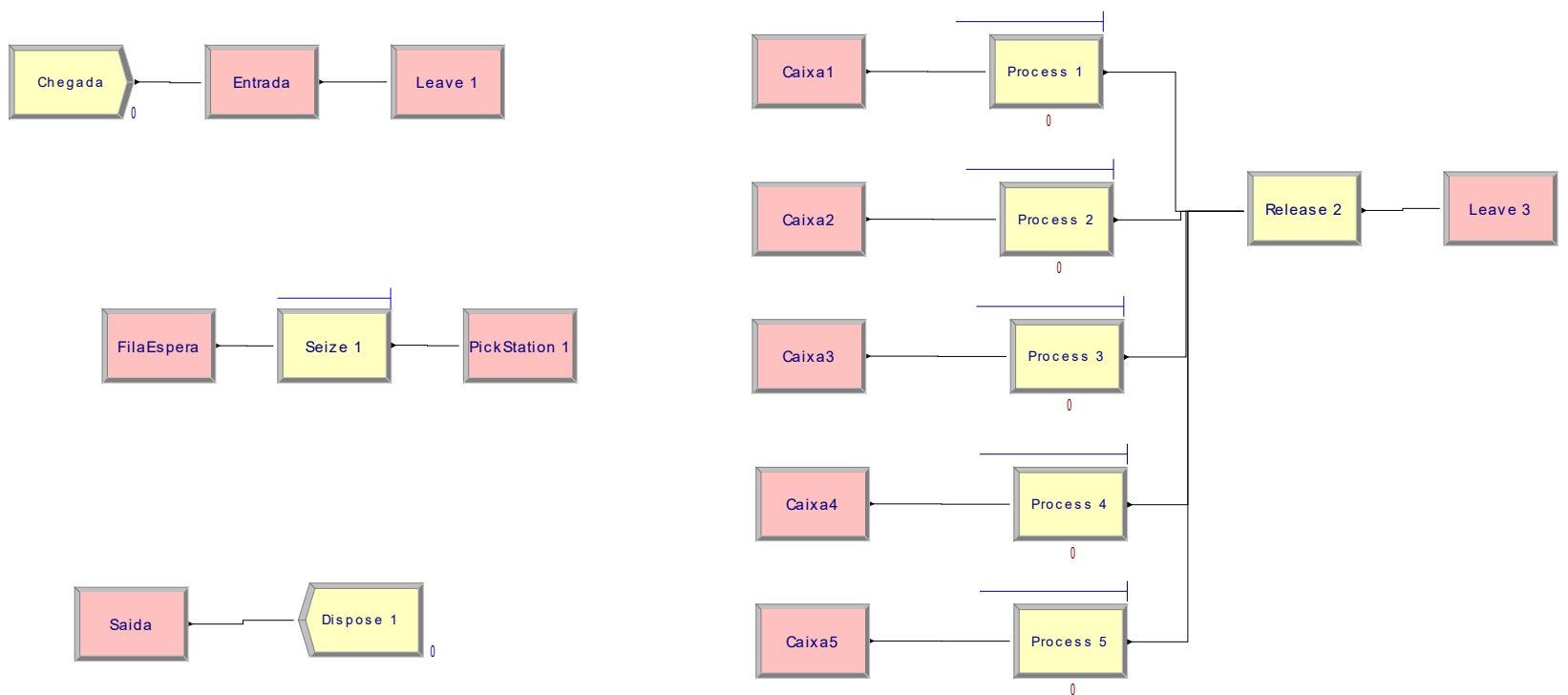

Figura 6 - Modelo Lógico do Sistema de Fila Única. Fonte: Simulador 
Figura 7

Modelo

\section{6:00:00}
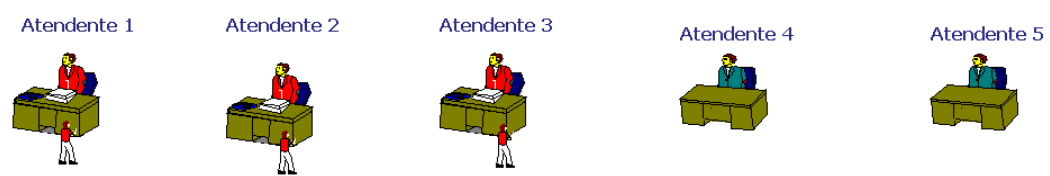
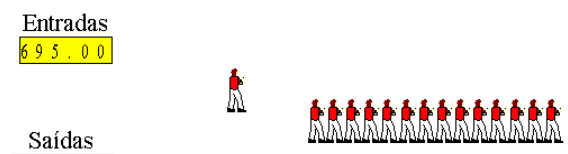

Animado do Sistema de Fila Única. Fonte: Simulador

O terceiro passo da metodologia é a interpretação dos relatórios gerados pelo Simulador Arena. O modelo foi rodado com uma replicação de 480 minutos, equivalente ao turno de trabalho da lotérica, utilizando as distribuições de probabilidades definidas anteriormente e o número de recursos existentes no estabelecimento.

De acordo com Chwif e Medina (2010) uma das formas de se validar um sistema e de se verificar um modelo é através da participação dos envolvidos diretamente com as atividades da organização junto com os projetistas. A validação do sistema foi feita na seção 4. O resultado da primeira simulação foi apresentado ao dono do estabelecimento e aos funcionários, confirmando que o modelo representa a realidade da lotérica. Os resultados obtidos pelo Programa Arena são diversos, todavia, neste artigo focaremos na análise dos tempos máximos de esperas dos dois modelos que são apresentados no gráfico1.

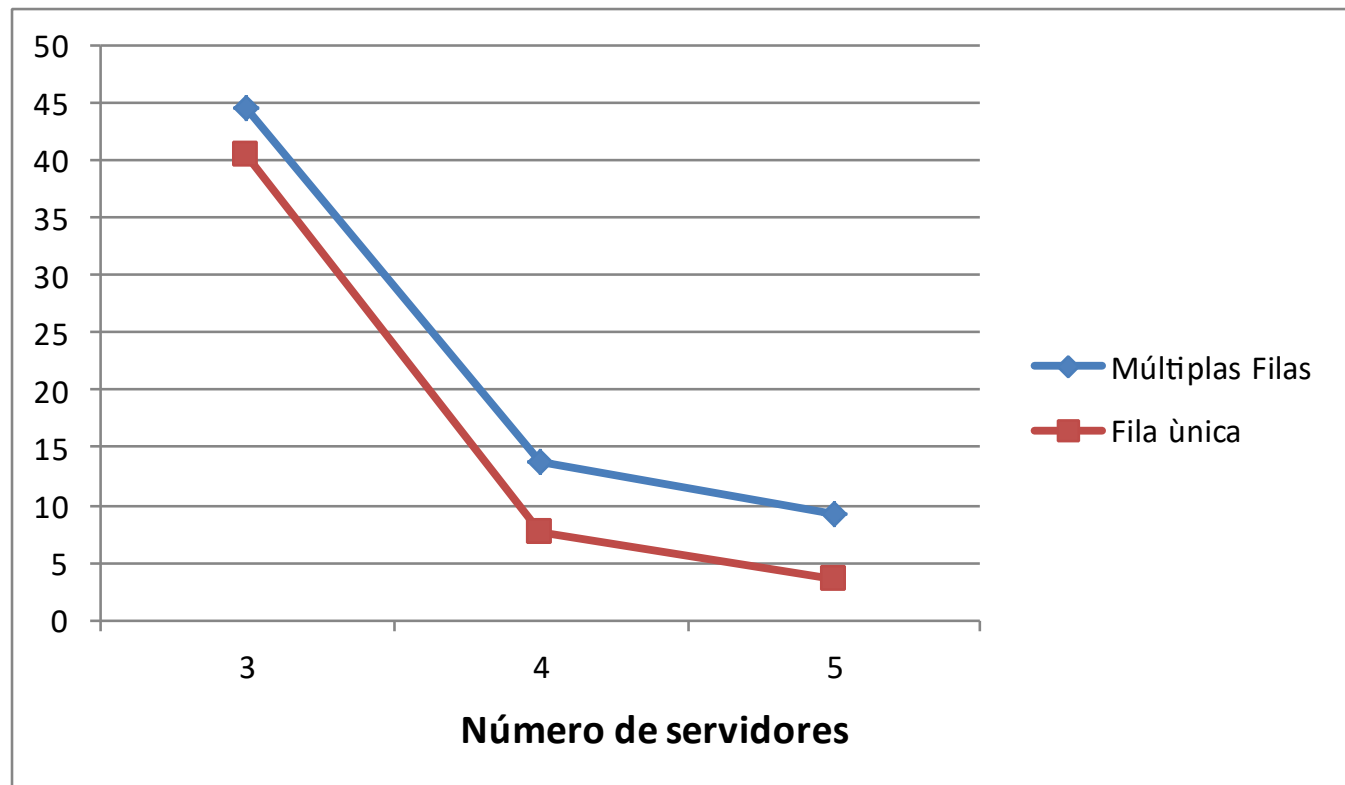

Filas.

Gráfico 1 - Tempo Máximo de Espera no Sistema de Fila Única e de Múltiplas

Analisando os resultados acima, constatamos que os dois sistemas de filas com três servidores não atendem as metas da organização, no período de maior demanda, pois o maior tempo de espera no sistema de fila única é de 40,51 minutos e o de filas múltiplas é de 44,61 
minutos. Com quatro servidores o modelo mostra que os tempos reduzem bastante. No sistema com fila única o cliente espera no máximo 7,52 minutos e no de múltiplas filas 13,64, atingindo a meta da organização. A utilização de cinco servidores é desnecessária, pois a variação é pouco expressiva se comparada com a mudança anterior. No sistema com múltiplas filas o maior tempo é de 9,15 minutos e no de fila única é de 3,57 minutos.

Também se constata que independente do número de servidores o sistema com fila única é melhor porque registrou o menor tempo de espera se comparado com o sistema de múltiplas filas. O sistema de fila única com três servidores tem um tempo máximo de espera $9 \%$ menor se compara com o de múltiplas filas. Com quatro servidores este percentual é bastante expressivo, pois equivale a $45 \%$.

\section{CONCLUSÃO}

Este trabalho mostrou que a Lotérica Loto Líder tem grandes desafios para prestar um serviço de qualidade, pois o longo tempo de espera nas filas causa transtornos aos usuários e prejuízos ao estabelecimento, visto que $10 \%$ desistem do atendimento.

A simulação mostrou-se uma técnica de fundamental importância para a tomada de decisão quanto ao número de caixas a serem alocados no período de maior demanda e ao tipo de sistema de filas a ser adotado sem implicar no risco de atuação direta no sistema. Os modelos desenvolvidos no software Arena mostraram que, no período crítico, o número ideal de atendentes é quatro. O sistema de fila única mostrou-se mais adequado, pois além de organizar melhor o espaço interno, o modelo registrou tempo máximo de espera menor comparado com o sistema de múltiplas filas.

Para evitar o custo de contratação de um novo funcionário, foi sugerido ao gestor o treinamento e alocação de um funcionário interno para o período de maior demanda. Também fica sugerido para trabalho futuro o estudo da demanda dos clientes preferenciais.

\section{REFERÊNCIAS}

ANDRADE, E. L. Introdução à pesquisa operacional. Rio de Janeiro, RJ: LTC, 1990.

CHWIF, L; \& MEDINA, A.C. Modelagem e Simulação de Eventos Discretos -

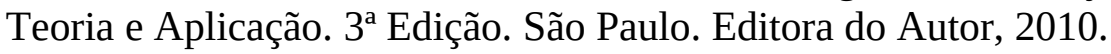

DA SILVA, V. KOZECHEN, A.P; FERREIRA, JEFESON; DE OLIVEIRA, G.D; MORAIS, M.F. Emprego da Simulação Computacional para Análise do Sistemas de Filas nos Caixas de um Supermercado. In: XXXVI Encontro Nacional de Engenharia de Produção, 2016, João Pessoa - PB. Anais do XXXVI ENEGEP.

DIAS, B.L; QUINTO, T; MORAES, C.F.S, MAGALHÃES, M.S. Análise da Fila de Acesso aos Micro-Ondas da Universidade Federal do Rio de Janeiro: Uma Proposta Baseada em um Modelo de Simulação. In XXIV Simpósio de Engenharia de Produção, 2017, Bauru - SP. Anais do XXIV SIMPEP.

FREITAS FILHO, P.J. Introdução à Modelagem e Simulação de Sistemas com Aplicações em Arena. $2^{a}$ Edição. Florianópolis - SC. Visual Books, 2008.

GIANESI, I.G; CORRÊA, H.L. Administração Estratégica de Serviços. São Paulo. Atlas, 1996. 
KELTON, W.D, LAW, A.M. Simulation Modeling and Analysis. 3rd ed. McGraw Hill, New York, 2000.

MAGALHÃES, M.S. Simulação do Sistema de Admissão de Emergência do Hospital Universitário Antônio Pedro. Dissertação de Mestrado em Engenharia de Produção, COPPE/UFRJ, Rio de Janeiro - RJ, 2006.

MAGALHÃES, M.S; MAGALHÃES, J.C; OLIVEIRA, I.F. Simulação do Processo de Produção de uma Pizzaria. In XXIV Simpósio de Engenharia de Produção, 2017, Bauru SP. Anais do XXIV SIMPEP.

MELO,H.R; LIMA F.R.S; MARSARO, M.S. Análise do Atendimento em uma Agência dos Correios por Meio da Teoria das Filas e da Simulação. In XXIV Simpósio de Engenharia de Produção, 2017, Bauru - SP. Anais do XXIV SIMPEP.

PEGDEN, C. D. Introduction to Simulation Using SIMAN - Second Edition. McGraw-Hill, 1995.

PETRÔNIO, G.M; LAUGENI, F.P. Administração da Produção. 2 2 ${ }^{\mathrm{a}}$ Edição revisada, ampliada e atual. São Paulo. Editora Saraiva 2005.

PRADO, D. S. Teoria das Filas e da Simulação. Ed. 5. Minas Gerais. Editora FALCONI, 2014.

PRADO, D. S. Usando o Arena em Simulação. Ed. 5. Minas Gerais. Editora FALCONI, 2014.

PRAIA, C.R; GOMES, C.F.S. Simulação Computacional Aplicada à Modelagem do Processo de Recebimento de Uniformes na Marinha do Brasil. In: X Simpósio de Excelência em Gestão e Tecnologia, 2013, Resende - RJ. Anais do X SEGeT.

PRODANOV, C.C; FREITAS E.C. Metodologia do Trabalho Científico: Método e Técnicas da Pesquisa e do Trabalho Acadêmico. 2. Edição, Novo Hamburgo, 2013. 277p. Disponível em <http://feevale.br/cultura/metodologia-do-trabalho-cientifico.> Acesso em 24/05/2017.

SLACK, N; CHAMBERS, S; JONHSTON, R. Administração da Produção $4^{\mathrm{a}}$ Edição. São Paulo. Editora Atlas, 2015.

YIN, ROBERT K. Estudo de Caso: planejamento e métodos. Edição 4. Tradução Ana Thorell. Editora Bookman. São Paulo, 2010. 